Deutscher Dialogmarketing Verband e.V. (Hrsg.)

Dialogmarketing Perspektiven 2010/2011 
GABLER RESEARCH 
Deutscher Dialogmarketing

Verband e.V. (Hrsg.)

\section{Dialogmarketing Perspektiven 2010/2011}

Tagungsband

5. wissenschaftlicher interdisziplinärer

Kongress für Dialogmarketing 
Bibliografische Information der Deutschen Nationalbibliothek

Die Deutsche Nationalbibliothek verzeichnet diese Publikation in der

Deutschen Nationalbibliografie; detaillierte bibliografische Daten sind im Internet über

$<$ http://dnb.d-nb.de> abrufbar.

1. Auflage 2011

Alle Rechte vorbehalten

(C) Gabler Verlag | Springer Fachmedien Wiesbaden GmbH 2011

Lektorat: Stefanie Brich | Ingrid Walther

Redaktion: Bettina Höfner

Gabler Verlag ist eine Marke von Springer Fachmedien.

Springer Fachmedien ist Teil der Fachverlagsgruppe Springer Science+Business Media. www.gabler.de

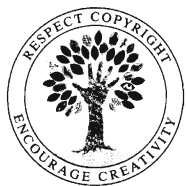

Das Werk einschließlich aller seiner Teile ist urheberrechtlich geschützt. Jede Verwertung außerhalb der engen Grenzen des Urheberrechtsgesetzes ist ohne Zustimmung des Verlags unzulässig und strafbar. Das gilt insbesondere für Vervielfältigungen, Übersetzungen, Mikroverfilmungen und die Einspeicherung und Verarbeitung in elektronischen Systemen.

Die Wiedergabe von Gebrauchsnamen, Handelsnamen, Warenbezeichnungen usw. in diesem Werk berechtigt auch ohne besondere Kennzeichnung nicht zu der Annahme, dass solche Namen im Sinne der Warenzeichen- und Markenschutz-Gesetzgebung als frei zu betrachten wären und daher von jedermann benutzt werden dürften.

Umschlaggestaltung: KünkelLopka Medienentwicklung, Heidelberg Gedruckt auf säurefreiem und chlorfrei gebleichtem Papier Printed in Germany

ISBN 978-3-8349-2754-5 


\section{Editorial}

\section{Tradition und Innovation}

Ein besonderes Jubiläum konnte der Deutsche Dialogmarketing Verband (DDV) im Oktober 2010 begehen: Zum 25. Mal wurde der Alfred Gerardi Gedächtnispreis, der wissenschaftliche Nachwuchspreis des DDV, vergeben. In den vergangenen 25 Jahren wurden damit knapp 100 Dissertationen, Diplom- und Abschlussarbeiten zu Themen des Dialogmarketings ausgezeichnet. Die Förderung der aktuellen wissenschaftlichen Auseinandersetzung mit dem Dialogmarketing ist damit eine seit einem Vierteljahrhundert bewährte und gelebte Tradition im DDV.

Deutlich jünger - aber auch schon „Tradition“ - ist der wissenschaftliche Kongress des DDV, in dessen Rahmen inzwischen die feierliche Preisverleihung des Alfred Gerardi Gedächtnispreises stattfindet. Der 5. wissenschaftliche interdisziplinäre Kongress für Dialogmarketing fand am 4. Oktober 2010 statt. Diese Konferenz, die eine einzigartige Plattform für den Erfahrungsaustausch zwischen Wissenschaft und Praxis bietet, konnte erfreulicherweise mit rund 120 Teilnehmern einen Besucherrekord verzeichnen. Unter der bewährten Tagungsleitung von Dr. Heinz Dallmer referierten und diskutierten Wissenschaftler und Praktiker einen Tag lang engagiert aktuelle Forschungsergebnisse und -thesen. Besonderheit des Kongresses ist hierbei der interdisziplinäre Ansatz: Wissenschaftler ganz unterschiedlicher Disziplinen tragen zur Diskussion bei und beleuchten die Themen aus unterschiedlichen Blickwinkeln. Wie schon in den vergangenen Jahren lag ein ausgeprägter Schwerpunkt der Tagung im Bereich Online-Medien insbesondere im Bereich Social Media sowie „Word-of-Mouth“. Fast alle Vorträge dieser Konferenz finden sich im vorliegenden Tagungsband vereint, in bereits bewährter Form ergänzt um weitere aktuelle Fachbeiträge.

Im Namen aller Mitglieder des DDV und stellvertretend für die Kongressteilnehmer sowie für die Leser dieses Tagungsbandes möchte ich mich ganz herzlich bedanken: Bei den Referenten und Autoren für die Tatsache, dass sie uns an ihren Forschungsergebnissen teilhaben lassen. Bei Dr. Heinz Dallmer für die kenntnisreiche Tagungsleitung des Kongresses. Und last but not least bei den Unternehmen und Organisationen, die mit einem großzügigen Sponsoring die Veranstaltung und die Realisierung dieses Tagungsbandes ermöglichten: Acxiom 
Deutschland GmbH, g k k DialogGroup GmbH, Jahns and Friends Agentur für Dialogmarketing und Werbung AG, JCG Consulting Group AG, MarAnCon Gesellschaft für Marketing, Analyse und Consulting mbH, Printus $\mathrm{GmbH}$, SAS Institute $\mathrm{GmbH}$, Schober Information Group, Siegfried Vögele Institut GmbH. Partner waren darüber hinaus der BVDW Bundesverband Digitale Wirtschaft, der Dialog Marketing Verband Österreich sowie der Schweizer Direktmarketing Verband. Als Medienpartner engagierten sich acquisa, marketingBÖRSE und OnetoOne.

Ein ganz besonderer Dank geht an Mary-Victoria Gerardi-Schmid und Hans R. Schmid, Printus GmbH, die den Alfred Gerardi Gedächtnispreis seit vielen Jahren fördern und den besonders festlichen Rahmen für den Kongress im Jubiläumsjahr des Wettbewerbs als Premiumsponsor ermöglicht haben.

Gerne würden wir den Dialog mit Ihnen fortsetzen: Der 6. wissenschaftliche interdisziplinäre Kongress für Dialogmarketing wird im Herbst 2011 stattfinden. Wir würden uns freuen, Sie dort begrüßen zu dürfen!

Martin Nitsche

DDV-Vizepräsident Bildung und Forschung

\section{Kontakt}

Martin Nitsche

Vizepräsident

Deutscher Dialogmarketing Verband e.V.

Hasengartenstraße 14

65189 Wiesbaden

info@ddv.de 


\section{Inhalt}

Editorial

Social Media Marketing - ein neues Marketing-Paradigma? 9

Michael H. Ceyp / Juhn-Petter Scupin

Word-of-Mouth Amplified -

An Exploration of Hotel Customer Feedback Websites

Petra Bouvain / Matthias Muskat / Birgit Muskat

Der Word-of-Mouth-Effekt als kalkulierbare Größe in der Mediaplanung.

Tina Anschütz / Ralph Sonntag

Welche Emotionen beeinflussen Konsumenten bei der Weiterleitung viraler Videospots?

Ellen Binggeser / Larissa Hammon / Stefan Hampel / Hajo Hippner

Herausforderungen im Social CRM und Mobile Business Rebecca Bulander

Herausforderungen an den Kundenservice durch Social MediaErgebnisse einer Befragung von Führungskräften aus der Callcenter-Branche 109 Heike Simmet

Dialogmarketing in Marktnischen aus Konsumentenperspektive

Tobias Schäfers / Markus Gräßler / Ronja Gresel

Informieren und Kaufen bei Multichannel-Retailern - empirische

Untersuchungen zum Verhalten der Endkunden

Hendrik Schröder / Annette Bohlmann / Matthias Witek / Silvia Zaharia 
Instrumente des Dialogmarketings im Maschinenbau

Larissa Greschuchna

Insights in der Versicherungsbranche -

Ergebnisse einer Mystery-Shopping-Studie

Ralf T. Kreutzer / Simone Spiegels

Vollautomatisches Predictive Targeting und Modellierung des

Realtime-Online-Verhaltens Andrea Ahlemeyer-Stubbe

Alfred Gerardi Gedächtnispreis 2010 227

Dank an die Sponsoren 231 